\title{
CAN GALACTIC HALOS BE MADE OF BARYONS?
}

\author{
Dennis J. HEGYI \\ Department of Physics, University of Michigan, Ann Arbor, MI, USA
}

and

\author{
Keith A. OLIVE \\ CERN, Geneva, Switzerland
}

Received 29 March 1983

\begin{abstract}
Several arguments are presented indicating that the apparently non-luminous matter forming massive halos of spiral galaxies is not baryonic. There are difficulties with a halo dominated by gas, snowballs, dust and rocks, jupiters, low mass stars, dead stars and neutron stars. Also, halos may not be composed of black holes unless they are either extremely efficiently accreting or primordial. Consequently, it appears that a significant fraction of the universe may be in the form of massive neutrinos, gravitinos, monopoles, etc.
\end{abstract}

There appears to be no escape from the conclusion that spiral galaxies are surrounded by a large amount of sub-luminous or non-luminous mass [1]; the conclusion is based on an application of simple newtonian mechanics to a system in equilibrium. The central issue at the present time concerns the nature of the halo mass. We have assembled several arguments pointing out the problems associated with baryonic halos thus suggesting that halos may not be made of baryons. These problems force one to seriously consider the possibility that halos which comprise a significant fraction of the mass of the universe, are either composed of massive neutrinos, photinos, gravitinos, etc., or of very efficiently accreting or primordial black holes.

The strongest evidence for the existence of massive galactic halos is based on the measured velocity of matter in stable circular orbit in the disks of spiral galaxies. The corresponding centripetal acceleration, calculated from the measured velocities, is larger than can be understood from the gravitational field arising from the visible galactic matter; additional matter, a massive halo, is postulated to explain the observed centripetal acceleration. Measurements $[2,3]$ on more than 50 spiral galaxies for which symmetric rotation curves have been obtained support the equilibrium condition, $M_{r}=(K / G) v^{2} r$ where $K$ is a constant of order unity, $G$ is the gravitational constant, $v$ is the observed rotational velocity, at galactic radius $r$, and $M_{r}$ is the mass contained within radius $r$. One would expect that if the bulk of the mass were contained in the luminous central regions, the rotational velocity would fall as $v \propto r^{-1 / 2}$. Instead, one finds that $v$ is independent of $r$ indicating that $M_{r}$ $\propto r$, or $\mathrm{d} M / \mathrm{d} r=$ const. Thus, there is an equal amount of mass per increment of galactic radius unlike the galaxy luminosity distribution which is centrally concentrated.

Other evidence supporting massive halos, though not as compelling as the galaxy rotation curves, includes binary galaxies $[4,5]$ and the stability of spiral arms [6]. Investigations $[7,8]$ pursuing independent directions have argued that the unseen mass is distributed spherically symmetrically.

Let us now explore the possibility that massive galactic halos are composed of baryons by considering a halo made of gas. Since we will require the halo to be both stable and static we assume that it must be in hydrostatic equilibrium, i.e., the halo is maintained only if it is at a temperature $T_{\text {eq }}$. For temperatures less than $T_{\text {eq }}$, the halo would collapse in a gravitational timescale $\tau_{\mathrm{c}}=(3 \pi / 32 \mathrm{G} \rho)^{1 / 2} \approx 5 \times 10^{8} \mathrm{yr}$, much less than the age of the galaxy. To determine $T_{\text {eq }}$, we use 
the following

$\mathrm{d} P_{r} / \mathrm{d} r=-G M_{r} \rho_{r} / r^{2}$

where $P_{r}$ and $\rho_{r}$ are the pressure and density, respectively, at radius $r$ and

$P_{r}=\left(2 \rho_{r} / m_{\mathrm{p}}\right) k T$

is the equation of state. Solving eqs. (2) and (3) for $T_{\text {eq }}$ with $M_{r} \propto r$ and $\rho_{r} \propto r^{-2}$ we have,

$T_{\text {eq }}=\left(G m_{\mathrm{p}} / 2 k\right)\left(M_{r} / r\right) \approx 1.9 \times 10^{6} \mathrm{~K}$

for $M_{r} \approx 10^{12} M_{\odot}$ at $r \approx 100 \mathrm{kpc}$.

A hot gas halo at temperature $T_{\text {eq }}$, however, would be emitting a thermal X-ray background which is in excess of the limits of the observed X-ray background. To see this, we compare the quantity $\delta C \Omega^{2}$, which is a measure of the X-ray emissivity of the halo matter evaluated at $T_{\text {eq }}$, with the observed upper limit derived from the X-ray background. Here $\delta \approx h_{0}^{3}$ where $h_{0}$ $=H_{0} /\left(100 \mathrm{~km} \mathrm{~s}^{-1} \mathrm{Mpc}^{-1}\right), C$ is a clumpiness parameter, and $\Omega=\rho / \rho_{\mathrm{c}}$. The quantity, $\rho$, is the average density of matter in the universe, $\rho_{\mathrm{H}}$ is the average halo density, $1.7 \times 10^{-26} \mathrm{~g} \mathrm{~cm}^{-3}$ corresponding to our standard halo containing $10^{12} M_{\odot}$ in $100 \mathrm{Kpc}$, and $\rho_{\mathrm{c}}$ $\equiv 3 H_{0}^{2} / 8 \pi G=1.88 \times 10^{-29} h_{0}^{2} \mathrm{~g} \mathrm{~cm}^{-3}$. To evaluate $\delta C \Omega^{2}$ for halos we use [1] $\Omega \geqslant \Omega_{\mathrm{H}}=[M / L]_{\text {spirals }} /$ $[M / L]_{\text {critical }} \geqslant 35 / 700=0.05$ and $C \Omega=\rho_{\mathrm{H}} / \rho_{\mathrm{c}}$ which follows directly from the definition, $C \equiv\left\langle\rho^{2}\right\rangle /\langle\rho\rangle^{2}$. With $h_{0}=\frac{1}{2}$ to obtain a lower limit on the halo X-ray emission, we find that for halos $\delta C \Omega^{2} \geqslant 22$. Silk [9], based on observations of the X-ray background, finds that at $T=2 \times 10^{6} \mathrm{~K}, \delta C \Omega^{2} \lesssim 1$. A halo of hot gas violates the upper limits by a factor of at least 20 .

Another possibility for the halo matter is low mass stars [10] or jupiters, that is, non-nuclear burning objects with mass $<0.08 M_{\odot}$. We shall show that if one uses a single power law for the distribution function describing the number of stars and jupiters of a given mass as a function of mass, where the index in the power law is chosen to match the observed stellar (nuclear burning) distribution function, such a halo will radiate much more light than is observed.

To draw this conclusion it is necessary to use an upper limit on the index, $x$, in the stellar mass distribution function which is defined by

$\phi(m)=A m^{-(1+x)}$,

where $\phi(m)$ is the number of stars per unit volume per unit mass. Constraints on $x$ which have been obtained from observation of narrow band spectral features [11] and stellar data in the solar neighborhood [12] for stars of mass $<0.8 M_{\odot}$ require $x \leqslant 1$ at the $2 \sigma$ level. Other data in the infrared $[13,14]$, though intrinsically less sensitive as a discriminant, are satisfied by the weaker constraint, $x \leqslant 1.35$.

Our conclusion is also sensitive to a second quantity, the smallest mass object which can gravitationally collapse, $m_{\min }$, also known as the Jeans' mass. Arguments on the fragmentation of gas clouds $[15-17]$ place a lower limit on the Jeans' mass of $0.007 M_{\odot}$ for a collapsing cloud consisting of hydrogen and helium. Because there are complications handling the radiative transfer in this mass range, we shall adopt $m_{\text {min }}$ $\geqslant 0.004 M_{\odot}$ since this limit was determined using an optically thin cloud which avoids these complications and should be a lower limit on $m_{\min }$ [18]. In fact, others argue [19] that the first stars to form may have masses $\gtrsim 1500 \mathrm{M}_{\odot}$.

We shall summarize the calculation of the surface brightness of the halo here and shall present the details elsewhere. Using the data of Tinsley and Gunn [11] for both dwarfs in the mass range $0.08 M_{\odot}$ to $0.8 M_{\odot}$ and for giants with $m_{\text {giant }}=0.8 M_{\odot}$ we have calculated the ratio of mass-to-luminosity in the $\mathrm{I}$ and $\mathrm{K}$ Johnson spectral bands for the halo of NGC 4565 . The rate of evolution of giants from the main sequence has been corrected for low metal abundance $\left(Z=10^{-5}\right)$ based on model calculations [20]. The calculated surface brightness must satisfy the observational data in the I and $\mathrm{K}$ band. The $2 \sigma$ lower limits on the mass-to-light ratio, $M / L$, for the halo of NGC 4565 expressed in solar units corresponding to the I data [21] and the $K$ data [22] are,

$M / L_{\mathrm{I}}>60 M_{\odot} / L_{\odot}, \mathrm{I}$

$M / L_{\mathrm{K}}>38 M_{\odot} / L_{\odot}, \dot{\mathrm{K}}$.

If we choose $m_{\min }=0.004 M_{\odot}$ and determine the power law index, $x$, necessary to satisfy the observational data, we find $x>1.6$ and $x>1.7$ for the $I$ and $\mathrm{K}$ bands, respectively.

Alternatively, rather than solve for a lower limit on $x$, we can choose $x$ and try to solve for $m_{\min }$. For $x$ $<1$, there is no solution for $m_{\min }$ consistent with the observations; it is not possible to put enough mass in the halo to satisfy the rotation curve and the surface 
brightness constraints. If we relax the constraint on $x$ and use the Salpeter value, $x=1.35$, we find that $x_{\min }$ must be less than $2 \times 10^{-4} M_{\odot}$, more than a factor of 20 below the calculated lower limit on $m_{\min }$. On this basis we find a halo filled with either low mass stars or jupiters inconsistent with present observations.

A recent observation [23] suggests that we are being much more conservative than may be necessary. It appears that the initial mass function turns over for $m$ $\gtrsim 0.1 M_{\odot}$. This result was based on stars with a metal abundance similar to that of the sun. For stars without metals, $m_{\mathrm{min}}$ is expected to be larger since metals allow a collapsing star to cool and fragment more effectively.

Next, we consider a halo composed of stars with an initial mass greater than $2 M_{\odot}$ which are now either white dwarfs or neutron stars. The maximum mass of white dwarfs is $\sim 1.4 M_{\odot}[24]$, which coincidentally, appears to be about equal to the mass of neutron stars. Because the two most precisely determined neutron star masses are the same to within the $1 \%$ measuring uncertainty [25], and because all neutron star mass determinations are consistent with $1.4 M_{\odot}$, we shall take this value for the mass of all neutron stars. Since stars with masses larger than $2 M_{\odot}$ must evolve to a remnant of only $1.4 M_{\odot}$, at least 40 percent of the present halo mass contained in these remnants must have been ejected. The problem with massive stars is to find a suitable place to hide their ejecta. The ejected mass cannot be in hot gas because of previous arguments and it cannot be in cool gas or it will quickly collapse into the disk. Depending on the exact distribution of stellar masses, there would be an additional problem. A significant amount [26] ( $\gtrsim 10 \%)$ of the ejected mass would be in the form of helium and metals.

One might expect an interstellar medium with metal in the halo to lead to a second generation of stars with a mass function similar to the solar neighborhood yielding a halo which is too bright. A second problem involves the contamination due to the ejected metals of the oldest stars in the galaxy. Observations show there exist old low mass stars in the nuclear bulge [27] with a metal abundance of $Z \sim 10^{-5}$. The metal abundance of these stars should be characteristic of the interstellar medium at the epoch when the stars formed. Unless the nuclear bulge formed substantially later than the massive halo (in which case the above arguments still apply) it is difficult to understand how the oldest stars would not be contaminated at a level which is larger than $Z \sim 10^{-5}$.

A similar argument applies to a halo composed primarily of metals, including rocks, dust or grains, etc. If the halo is composed primarily of metals then $\Omega_{\text {metals }} \geqslant 0.05$. Since the mass in the disk and nuclear bulge of the galaxy corresponds to an $\Omega \sim 10^{-3}$, then it would be impossible to find stars with a metal abundance as small as $Z \sim 10^{-5}$ if more than one part in $5 \times 10^{6}$ of the halo material contaminated the first generation of stars. The above arguments for the evolution of stars with initial masses greater than $2 M_{\odot}$ also rule out a halo of neutron stars for the same reasons.

We now consider a halo composed of snowballs, objects consisting primarily of condensed hydrogen which are held together by electrostatic forces. Snowballs, consequently, are not ruled out by arguments based on the Jeans' mass which only involve gravitational bind. ing. We shall show, nevertheless, that though we cannot envision any way in which snowballs might form, they would rapidly evaporate into a gaseous state, if they did form.

For our standard halo, $10^{12} M_{\odot}$ in $100 \mathrm{Kpc}$ radius, snowballs of density equal to that of solid hydrogen, $\rho_{\mathrm{s}} \sim 7 \times 10^{-2} \mathrm{~g} \mathrm{~cm}^{-3}$, must have a radius greater than $1 \mathrm{~cm}$ to avoid all collisions during their lifetime, $10^{10}$ yr. At typical halo velocities $\sim 250 \mathrm{~km} \mathrm{~s}^{-1}$ or $500 \mathrm{eV}$ per hydrogen atom, kinetic energies are so much larger than typical binding energies, $\sim 1 \mathrm{eV}$, that a single collision would destroy a snowball. Consequently, under such conditions, they must form without dissipation at a density about equal to the present halo density, $\rho_{\mathrm{H}} \sim 1.7 \times 10^{-26} \mathrm{~g} \mathrm{~cm}^{-3}$ in a timescale $\tau_{\mathrm{c}} \sim(3 \pi /$ $\left.32 G \rho_{\mathrm{H}}\right)^{1 / 2}$.

The snowballs will be most stable against evaporation in a universe with the lowest temperature cosmic background radiation. Assuming that $\Omega \leqslant 2$, this will occur with $\Omega=2$ and $h_{0}=1$. Because of the lack of dissipation during the formation of snowballs, they must form when the universe has an age equal to $2 \tau_{\mathrm{c}}$. Under these most favorable conditions the temperature of the cosmic background radiation is $\sim 7.6 \mathrm{~K}$. At this temperature, in equilibrium, the vapor pressure of solid hydrogen is $0.045 \mathrm{~mm}$ [28]. This is sufficiently high so that there is no equilibrium between the gaseous and solid phases of hydrogen; all the hydrogen is in the gaseous state. It may be shown using standard two phase thermodynamic equilibrium methods [29] that, in fact, 
the halo density would have to increase by a factor $\sim 10^{19}$ before any solid hydrogen could remain in equilibrium with the gas.

Having established the ultimate equilibrium state, we consider the rate at which evaporation takes place. The time for evaporation of an $\mathrm{H}_{2}$ molecule, $t_{\mathrm{ev}}$, should be [30]

$t_{\mathrm{ev}} \sim \nu_{0}^{-1} \exp (b / k T)$.

where $\nu_{0} \sim 10^{12} \mathrm{~s}^{-1}$ is the characteristic vibration frequency of a solid hydrogen lattice and $b$ is the binding energy of a hydrogen molecule to the surface of the solid. It is possible to estimate $b$ by using the principle of corresponding states [31]. For a Lennard Jones potential the depth of the well, which we shall use as an approximate value of $b$, is, $b \sim 0.77 T_{\mathrm{c}} k . T_{\mathrm{c}}$ is the critical temperature, equal to $33.3 \mathrm{~K}$ for $\mathrm{H}_{2}$ and $k$ is Boltzmann's constant. With these numbers, $t_{\mathrm{ev}}$ $\sim 10^{-11} \mathrm{~s}$, so we may conclude that evaporation proceeds rapidly. It is also possible to show that snowballs would evaporate at $2.7 \mathrm{~K}$.

The final possibility is that the halo is composed of black holes. While it is not possible to rule them out, particularly massive black holes with masses larger than $10^{2} M_{\odot}$, some general statements can be made. Consistent with our argument, however, we point out though black holes are certainly of interest here, they have no well defined baryon number.

The initial mass function which evolved into a halo of black holes cannot have had many low mass stars $\left(<1 M_{\odot}\right)$; otherwise, the stars would be observable today. One might expect a halo composed of black holes of mass $\sim 10 M_{\odot}$ to have evolved from similar mass stars. But since the evolution of stars in this mass range appears to reliably lead to mass loss, black holes in this mass range are not favored. However, any black hole which can very efficiently accrete its ejecta remains a possibility. While this may not appear likely for low mass black holes, no such statement can be made about supermassive black holes because so little is known about them. Also, it is not possible to rule out primordial black holes.

In conclusion, we have argued that there may be significant amounts of stable non-baryonic matter on galactic scales. In addition, it does not appear likely that the halo is composed of black holes, unless they are very efficiently accreting or primordial. Consequently, other constituents for the halo should be seriously considered. Popular suggestions include massive neutrinos $[32,33]$ and, from supersymmetric theories, massive gravitinos [34] or photinos. Depending on their type and velocity, magnetic monopoles could provide the halo mass; however, because of the constraints on monopoles [35-39], they appear to be less likely.

We would like to thank J. Ellis, G.W. Ford, F. Palla and D. Schramm for helpful discussions.

\section{References}

[1] S.M. Faber and J.S. Gallagher, Ann. Rev. Astron. Astrophys. 17 (1979) 135.

[2] N. Krumm and E.E. Salpeter, Astron om. J. 84 (1979) 1138.

[3] V.C. Rubin, W.K. Ford Jr. and N. Thonnard, Astrophys. J. 225 (1978) L 107.

[4] E.L. Turner, Astrophys. J. 208 (1976) 304.

[5] S.D. Peterson, Astrophys. J. 232 (1979) 20.

[6] J.P. Ostriker and P.J.E. Peebles, Astrophys. J. 186 (1973) 467.

[7] E.M. Saar, in: IAU Symposium 84, The large-scale characteristics of the galaxy, ed. W.B. Burton (1978).

[8] D.G. Monet, D.O. Richstone and P.L. Schechter, Astrophys. J. 245 (1981) 454.

[9] J. Silk, Ann. Rev. Astron. Astrophys. 11 (1973) 269.

[10] A. Dekel and J. Shaham, Astron. Astrophys. 74 (1979) 186.

[11] B.M. Tinsley and J.E. Gunn, Astrophys. J. 203 (1976) 52.

[12] G.E. Miller and J.M. Scalo, Astrophys. J. Suppl. 41 (1979) 513.

[13] M. Aaronson, J.G. Cohen, J. Mould and M. Malkan, Astrophys. J. 223 (1978) 824.

[14] J.A. Frogel, S.E. Persson and J.G. Cohen, Astrophys. J. 240 (1980) 785.

[15] C. Low and D. Lynden-Bell, Mon. Not. R. Astron, Soc. 176 (1976) 367.

[16] M.J. Rees, Mon. Not. R. Astron. Soc. 176 (1976) 483.

[17] J. Silk, Astrophys. J. 256 (1982) 514.

[18] F. Palla, E.E. Salpeter and S.W. Stahler, preprint.

[19] J.F. Tohline, Astrophys. J. 239 (1980) 417.

[20] A.V. Sweigart and P.G. Gross, Astrophys. J. Suppl. 36 (1978) 405.

[21] D.J. Hegyi, in: Proc. Moriond Astrophysics Meeting, eds. J. Audouze et al. (Frontieres, Oreux, 1981).

[22] S.P. Boughn, P.R. Saulson and M. Seldner, Astrophys. J. 250 (1981) L15.

[23] R.G. Probst and R.W. O'Connell, Astrophys. J. 252 (1982) L69.

[24] S. Chandrasekhar, Mon. Not. R. Astron. Soc. 95 (1935) 207.

[25] J.H. Taylor and J.M. Weisberg, Astrophys. J. 253 (1982) 908. 
[26] W.D. Arnett, Astrophys. J. 219 (1978) 1008.

[27] H.E. Bond, Astrophys. J. 248 (1981) 606.

[28] V.J. Johnson, A. compendium of the properties of materials at low temperature (Phase I) U.S. Air Force (1960).

[29] See, e.g. F.W. Sears, An introduction to thermodynamics, the kinetic theory of gases and statistical mechanics (Addison-Wesley, Reading, MA, 1950) Ch. 6.

[30] D. Hollenbach and E.E. Salpeter, Astrophys. J. 163 (1971) 155.

[31] J.O. Hirschfelder, C.F. Curtiss and R.B. Bird, Molecular theory of gases and liquids (Wiley, New York, 1954) Ch. 4.

[32] A.L. Melott, Nature 296 (1982) 721.
[33] J.R. Bond and A.S. Szalay, Proc. Neutrino 81 (Hawaii, 1981) p. 59.

[34] H. Pagels and J.R. Primack, Phys. Rev. Lett. 48 (1982) 223.

[35] E.N. Parker, Astrophys. J. 160 (1960) 383.

[36] M.S. Turner, E.N. Parker and J.T. Bogban, Phys. Rev. D. 26 (1982) 1296

[37] E.W. Kolb, S.A. Colgate and J.A. Harvey, Phys. Rev. Lett. 49 (1982) 1373.

[38] J. Ellis. D.V. Nanopoulos and K.A. Olive, Phys. Lett. 116B (1982) 127.

[39] F.A. Bais, J. Ellis, D.V. Nanopoulos and K.A. Olive, CERN preprint TH-3383. 